FORMATION Formation emploi

Revue française de sciences sociales

123 | Juillet-Septembre 2013

Pêle-mêle

\title{
Les femmes de chambre de l'hôtellerie de luxe ou le déclassement d'une élite invisible
}

Chambermaids in luxury hotels. The fall in social status of an invisible elite Die Zimmermädchen von Luxushotels: Herabstufung einer unsichtbaren Elite Las camareras de la hoteleria de lujo : el desclasmiento de una élite invisible

\section{Pascal Guibert, Gilles Lazuech et Vincent Troger}

\section{(2) OpenEdition}

Journals

\section{Édition électronique}

URL : https://journals.openedition.org/formationemploi/4035

DOI : 10.4000/formationemploi.4035

ISSN : 2107-0946

\section{Éditeur}

La Documentation française

\section{Édition imprimée}

Date de publication : 30 septembre 2013

Pagination : $27-44$

ISSN : 0759-6340

\section{Référence électronique}

Pascal Guibert, Gilles Lazuech et Vincent Troger, « Les femmes de chambre de l'hôtellerie de luxe ou le déclassement d'une élite invisible », Formation emploi [En ligne], 123 | Juillet-Septembre 2013, mis en ligne le 07 octobre 2015, consulté le 21 septembre 2021. URL : http://journals.openedition.org/ formationemploi/4035; DOI : https://doi.org/10.4000/formationemploi.4035 


\title{
Les femmes de chambre de l'hôtellerie de luxe ou le déclassement d'une élite invisible
}

\begin{abstract}
PASCAL GUIBERT
Maître de conférences à l'université de Nantes au département de sciences de l'éducation. II est membre du Centre de recherche sur l'éducation de Nantes (CREN, EA2661).
\end{abstract}

GILLES LAZUECH Maître de conférences habilité à diriger des recherches à l'UFR (unité de formation et de recherches) de sociologie de Nantes.

VINCENT TROGER Maître de conférences en sciences de l'éducation, Centre de recherche sur l'éducation de Nantes (CREN).

Résumé

Les femmes de chambre de I'hôtellerie de luxe ou le déclassement d'uné élite invisible

Les femmes de chambre de l'hôtellerie de luxe (palaces et hôtels 4 étoiles) constituaient une élite préservée au sein d'un métier socialement dévalorisé. Les observations de terrain montrent que si les conditions de travail sont objectivement meilleures que dans tous les autres secteurs de l'hôtellerie, les transformations managériales et l'évolution de la clientèle ont mis un terme à la "relation enchantée " que ces personnels entretenaient avec des clients héritiers de valeurs aristocratiques, ainsi qu'aux avantages matériels et symboliques qui y étaient associés.

Mots clés : Métier de l'hôtellerie-restauration, Conditions de travail

Abstract

Chambermaids in luxury hotels. The fall in social status of an invisible elite

Traditionally, chambermaids employed in four-star hotels and luxury palaces have been considered as an elite exception within an otherwise socially depreciated profession. Field work has demonstrated that while their actual working conditions are objectively better than those found in other areas of the hotel industry, changes in managing 
techniques and expectations of the clientele have put an end to any "privileged relationship" that may have existed in the past. A relationship that was closely linked to an aristocratic value system based on symbolic and material advantages.

Keywords: occupation in hotel and catering, working condition

Journal of Economic Literature: $\mathbf{J} 8 \mathbf{1}$

Traduction : Auteurs

Nous avons commencé cette recherche en partant de l'hypothèse que les femmes de chambre travaillant dans l'hôtellerie de luxe se trouvent dans une situation professionnelle remarquable. Au sein des métiers du nettoyage et du ménage qui sont parmi les plus socialement dévalorisés, elles apparaissent comme une élite professionnelle ; en effet, comme nous le verrons plus loin, les femmes de chambre rencontrées dans l'hôtellerie de luxe jouissent de conditions de travail qui les distinguent des personnels d'entretien de l'hôtellerie standard et qui les éloignent des codes comportementaux des catégories populaires.

En effet, les salariés de ce segment des personnels de l'hôtellerie exercent leur métier dans un cadre d'exception, servent une clientèle exigeante et nous semblaient pour cela devoir mobiliser un niveau de compétences techniques et relationnelles très élevé. Ces personnels sont aussi réputés pour bénéficier, dans ces établissements prestigieux, de rémunérations gratifiantes, notamment grâce aux pourboires. Par ailleurs, contrairement à d'autres professions valorisées, cette élite est invisible. D'une part parce que le travail d'entretien et de propreté, lorsqu'il est réussi, consiste précisément à rendre invisibles les raisons pour lesquelles ce travail a été exécuté (Vigarello 1985), et d'autre part, parce que ceux qui l'exécutent ont pour consigne d'être très discrets, ce qui les oblige à se rendre aussi invisibles que possible aux yeux de la clientèle (Guégnard, Mériot, 2009).

Au fur et à mesure de notre exploration, nous avons compris que si cette hypothèse n'était pas dénuée de pertinence, elle reposait sur des représentations partiellement anachroniques des activités de l'hôtellerie de luxe. Nous avons en effet rencontré un personnel porteur d'un discours de déploration du passé, de regret d'un temps où les conditions de travail auraient été plus gratifiantes et valorisantes. Il s'est avéré que ce discours, s'il n'est évidemment pas exempt de la part de subjectivité propre à tous les acteurs sociaux, s'ancre néanmoins dans la réalité objective d'une transformation des conditions de travail, induite à la fois par l'émergence de nouvelles pratiques managériales et par l'arrivée, dans l'hôtellerie de luxe, d'une nouvelle clientèle. C'est pour- 
quoi il nous est progressivement apparu que l'hypothèse d'un possible déclassement du métier était plus pertinente pour en comprendre les évolutions récentes.

La première partie de l'article met donc l'accent sur les conditions de travail objectivement favorables dont bénéficient encore ces personnels d'entretien. La seconde partie analyse les conséquences de la transformation de la clientèle et des pratiques managériales sur le travail des personnels interrogés. S’ils ont pu, dans le passé, construire un sentiment d'appartenance à une élite professionnelle, on peut désormais identifier les raisons objectives et subjectives d'un sentiment inverse de banalisation, voire de déclassement.

\section{Encadré 1}

\section{Terrain d'enquête : L'hôtellerie de luxe}

La classification « hôtellerie de luxe » regroupe usuellement deux catégories d'établissements : les hôtels « haut de gamme » (4 étoiles) et « très haut de gamme » (5 étoiles, dont les palaces). Le terrain d'enquête est constitué de deux palaces ${ }^{(1)}$ et de deux hôtels de chaînes 4 étoiles dit " gros porteurs » (2) et représentatifs de chacune de ces deux catégories en France.

Les premiers, qui s'adressent à une clientèle très fortunée, ont construit leur réputation sur l'exceptionnalité des lieux et des décors ainsi que sur les services qu'ils offrent à leurs clients. Ces établissements d'exception ont été conçus selon le modèle de la Maison bourgeoise ${ }^{(3)} \mathrm{dont}$ ils sont un prolongement (Pinçon, Pinçon-Charlot 1996). Dans ces établissements, un registre particulier (le cardex) consigne les habitudes des clients habituels afin de pouvoir anticiper leurs attentes $\left({ }^{4}\right.$.

Les palaces offrent aussi la possibilité de retrouver, même après plusieurs mois d'absence, leur suite dans l'état exact ou ils l'avaient laissée lors de leur départ. Ce service, appelé « la réserve», est obtenu en photographiant chaque pièce de manière à restituer à sa place chaque objet laissé par le client lors de son départ.

Les établissements dits " gros porteurs » touchent une clientèle aisée mais socialement moins exceptionnelle que la précédente. Ces établissements appartiennent à des grands groupes hôteliers de dimension internationale et ils ont construit leur réputation sur des critères de confort standardisés à l'échelle mondiale selon la stratégie du marketing de la marque. Dans ces hôtels, qui comptent entre 900 et 1000 chambres, la clientèle ne s'installe pas et il n'y a pas une prise en charge personnalisée du client. Le côté " maison » s'efface donc au profit de celui de " chaîne haut de gamme ». Les différences entre les deux catégories d'établissement ressortent très nettement lorsque l'on observe les chambres mises à disposition des clients. Dans le cas des palaces, chacune des chambres (et des suites) bénéficie d'un ameublement et d'une décoration unique, alors que dans les " gros porteurs » le mobilier est standard.

(1) : En France, seulement 8 établissements ont obtenu le label " palace » en 2010. Ce label, décerné par le secrétariat d'Etat au Tourisme, vise à distinguer des établissements d'exception des autres hôtels de luxe. A Paris, les établissements distingués sont Le Bristol, le Meurice, le Park Hyatt Paris-Vendôme et le Plaza-Athénée. En régions, l'hôtel du Palais à Biarritz, les Airelles et Cheval Blanc à Courchevel et le Grand Hôtel Cap Ferrat à Saint-Jean-Cap-Ferrat. 


\section{Encadré 1 (Suite)}

(2) : La désignation « gros porteurs » est celle habituellement utilisée par les professionnels du secteur de l'hôtellerie. Les deux établissements dans lesquels nous avons enquêté ont été créés au début des années 70 par une grande compagnie aérienne. II s'agissait de proposer à des cadres étrangers en déplacement, outre le transport aérien, une offre d'hébergement et de restauration, ainsi que d'offrir à de grandes entreprises un espace propice à la tenue de séminaires. Depuis 2005, cette chaîne d'origine française a été rachetée par un groupe hôtelier américain disposant de plus de 120 établissements dans le monde.

(3) : Traditionnellement, ce modèle se caractérisait par l'emploi de " gens de maison » qui assuraient un service domestique adapté aux besoins particuliers de la famille dans le cadre d'une relation de proximité. Cette terminologie est encore en usage dans un palace parisien pour qualifier une emploi de cadre (« maîtresse de maison »), de même que celui de « gouvernante » qui est demeuré quant à lui d'usage courant.

(4) : Ainsi les services concernés savent que tel client n'aime que l'eau de Badoit, que tel autre ne supporte pas les savonnettes Hermès ou encore que celui-là affectionne particulièrement les roses rouges, etc. 


\section{Encadré 2}

\section{Méthodologie}

Plusieurs mois avant que nous puissions effectuer nos entretiens, nous avons été reçus dans chacun des établissements par des membres de la direction générale. L'accord initial des directions s'est fondé sur l'idée que notre présence pouvait être un outil de valorisation pour un personnel subalterne dont la qualité du travail est indispensable au succès d'un service produit tendant à valoriser l'excellence. Il s'est construit aussi sur le sentiment que la restitution des propos échangés lors des entretiens pouvait être utile à la direction des ressources humaines dans un contexte de modification des procédures d'exécution du travail et des modalités de recrutement de leurs personnels d'étage.

Notre enquête a été effectuée en 2010 par observations et par entretiens. Les observations ont été menées dans chacun des quatre établissements par immersion d'une à deux journées pendant lesquelles l'ensemble du travail des personnels d'étage a été observé : entretien des chambres, mise en place de la décoration, planification du travail, ainsi que partage des temps de pause et de restauration, etc. Les 28 entretiens individuels ont été menés, tous sur le lieu de travail. Ils se répartissent de la façon suivante :

\begin{tabular}{|c|c|c|c|}
\hline & & Palaces & Hôtels 4 étoiles \\
\hline \multirow{2}{*}{ Femmes de chambre et Valets } & Ancienneté forte (Plus de 15 ans) & 4 & 5 \\
\hline & Ancienneté faible (moins de 5 ans) & 2 & 5 \\
\hline \multicolumn{2}{|l|}{ Gouvernantes } & 4 & 4 \\
\hline \multicolumn{2}{|l|}{ Gouvernantes générales } & 1 & 2 \\
\hline \multicolumn{2}{|l|}{ DRH } & & 1 \\
\hline \multicolumn{2}{|l|}{ Total } & 11 & 17 \\
\hline
\end{tabular}

Nous avons constitué le corpus d'entretiens en prenant en considération les critères suivants : centrer l'enquête sur les femmes; s'assurer d'un nombre significatif de salariés recrutés depuis 15 à 30 ans et de salariés ayant encore un statut d'intérimaire ; avoir des délégués du personnel et/ou des représentants syndicaux. Enfin, des entretiens ont été réalisés avec les personnels d'encadrement direct des femmes de chambre : gouvernantes d'étage et gouvernantes générales, responsables des ressources humaines.

Les entretiens non directifs ont été effectués sur la base du volontariat. D'une durée moyenne d'une heure trente, ils ont porté sur la formation, l'accès à l'emploi, les conditions de travail, les relations avec les collègues et les évolutions de carrière et ont été analysés à l'aune des observations recueillies selon une démarche de type compréhensive et empirique (Becker, 2002). 


\section{La noblesse de la profession}

De nombreux travaux ont été consacrés aux élites professionnelles au sein des métiers d'exécution. Ces travaux, qui utilisent parfois la notion d'" aristocratie ", voire d'« avantgarde ", mettent en avant certaines caractéristiques singulières de ces professionnels comme, par exemple, l'importance de la formation initiale ou continue, les savoir-faire liés au métier, l'identité professionnelle construite sur un passé commun, les capacités à la mobilisation et à la résistance qui les distinguent des autres salariés (Charle 1987, Pigenet, 1990). Les recherches plus récentes qui sont consacrées aux salariés à statut prolongent pour partie les travaux menés à propos des "petites» élites (Cartier, Retière, Siblot 2010) : dans un contexte de fragilisation des conditions d'emploi, ceux qui bénéficient d'un emploi à statut finiraient par constituer une sorte d'îlot protégé comparativement à l'augmentation des emplois précaires, dont la proportion est, dans le secteur de l'hôtellerie, particulièrement élevée (Guégnard, 2004 ; Guégnard, Mériot, 2007).

Les femmes de chambres et les valets ${ }^{1}$ qui ont été recrutés au cours des années 80 et 90 dans les établissements de luxe constituent incontestablement, selon ces critères, une élite au sein des professionnels du nettoyage. Ces personnels, recrutés sur la base de la reconnaissance par leur hiérarchie de compétences professionnelles spécifiques, bénéficient de conditions de travail favorables ${ }^{2}$. Ils disposent d'un contrat de travail à durée indéterminée, ont la possibilité de bénéficier d'une structure syndicale active dans les établissements et bénéficient d'avantages divers (comme par exemple un comité d'entreprise).

Ce sont ces conditions de travail protectrices que nous mettons dans un premier temps en évidence, avant d'observer ensuite comment ces privilèges relatifs se manifestent au travers de pratiques professionnelles de distinction.

\subsection{Un cadre de travail protégé}

L'environnement dans lequel ces personnels travaillent se distingue par sa qualité de confort et de décoration. Pour l'observateur peu habitué, pénétrer dans un palace ne laisse pas indifférent, ne serait-ce que par la qualité et le raffinement du mobilier, des revêtements de sols, des éclairages, des objets de décoration, etc. Ainsi, le contexte de travail des salariés de l'hôtellerie de luxe se distingue très nettement de celui des établissements standards. Même si cela ne change pas fondamentalement la nature d'un travail qui reste

\footnotetext{
1 Valet de chambre est le masculin de femme de chambre. Appelés aussi équipiers, ils effectuent les travaux importants : nettoyage des moquette, manutention du mobilier, du linge,...

2 Dans le secteur de l'hôtellerie, les plages horaires de travail constituent un enjeu majeur de distinction entre les salariés. Les femmes de chambre ayant le plus d'ancienneté dans l'établissement peuvent choisir les horaires et les jours de travail qui leur conviennent mieux, alors que ce n'est pas le cas pour le personnel intérimaire.
} 
principalement celui du nettoyage, l'effet qu'il produit n'est ni superficiel ni éphémère. Il crée une sensation de qualité de vie et de confort que la plupart des salariés que nous avons rencontrés ont tenu à nous exprimer : "Ça fait luxe, c'est le luxe quand tu travailles dans des endroits comme ça. " (femme de chambre, 35 ans, hôtel de chaîne). "C'est beau, on travaille dans le beau et ça c'est irremplaçable "(femme de chambre, 47 ans, palace)

Ensuite, les femmes de chambre en CDI (contrat à durée indéterminée) à plein temps, ayant entre 15 et 30 ans d'ancienneté, perçoivent un salaire qui s'échelonnait, en 2010, de $1400 €$ à $1600 €$ nets (le SMIC - salaire minimum interprofessionnel de croissance net était à cette date légèrement inférieur à $1100 €$ pour un emploi à temps plein). Les rémunérations sont plus élevées dans les palaces où elles peuvent atteindre $1800 €$ hors prime. A cette rémunération de base s'ajoutent diverses primes pouvant représenter au moins la valeur d'un $13^{\mathrm{e}}$ mois de salaire ${ }^{3}$.

Outre le salaire et les primes, le pourboire est la troisième dimension constitutive de la rémunération. Il est plus difficile de les évaluer tant le sujet demeure tabou. L'ensemble des personnels rencontrés a cependant été unanime pour constater une baisse significative de ces gratifications depuis une dizaine d'années. Dans l'un des deux hôtels de chaîne, où la clientèle est socialement moins prestigieuse, les femmes de chambre ont évoqué des pourboires très modestes (autour d'une dizaine d'euros par semaine). Si la situation actuelle n'a plus rien à voir avec ce qui se pratiquait il y a encore quelques dizaines d'années, là encore, travailler dans un palace semble plus intéressant puisque certaines femmes de chambre nous ont confié toucher jusqu’à 100 euros de pourboire par semaine.

Si l'on prend pour critères d'appartenance à une élite professionnelle les conditions de travail et le statut de l'emploi (Castel, 1995), on doit relever dans ces établissements un niveau de "garanties " assez inédit comparativement à ce qui s'observe dans les autres établissements de l'hôtellerie. Ces garanties concernent principalement une plus grande sécurité d'emploi que dans l'hôtellerie, la présence de CE, des possibilités de formation continue, un Comité d'Hygiène, de Sécurité et des Conditions de Travail (CHSCT), ainsi qu'une plus grande souplesse dans le choix des emplois du temps pour les femmes de chambre titulaires.

Ainsi les grands hôtels comme les palaces sont des grandes PME (petites et moyenns entreprises) (plus de 300 salariés), alors que dans ce secteur, $80 \%$ des entreprises ont moins de 10 salariés (INSEE, 2003). Le personnel d'étages en CDI (contrat à durée déterminée) représente plus d'une cinquantaine de personnes (plus de 100 tous statuts confondus). Ce personnel est donc suffisamment nombreux pour autoriser l'existence de sections syndicales et de délégués du personnel en mesure d'imposer un rapport de force moins défavo-

\footnotetext{
3 Ces primes sont beaucoup moins avantageuses que l'ancien système qui associait les femmes de chambres au Chiffre d'Affaires réalisé par l'hôtel (Loi Godard). L'introduction des primes (en particulier de primes conditionnées à la réalisation d'objectifs quantitatifs) marque d'ailleurs le moment précis d'une «transformation » du métier ,du moins dans la mémoire des salariés.
} 
rable aux salariés que dans le reste de la profession : "J'ai fait un procès à l'hôtel parce que c'était mon droit, (...) ils ont fait appel et j'ai gagné l'appel, jai eu mon augmentation (...) je suis syndiquée. J'ai fait un procès parce que c'est mon droit et ils mont donné ce que je voulais." (femme de chambre, 42 ans, hôtel de chaîne). "Il y a 6 ans on a fait une grève de 19 jours (...) Avec le DRH c'était plus serré, sauf que ce monsieur il a été obligé de discuter parce quion est descendu dans le grand hall, on était au moins 60 (...). On a dit au DRH 'on veut pas parler avec vous, faites descendre le directeur' " (femme de chambre, 37 ans, hôtel de chaîne)

Pour les mêmes raisons d'ordre légal, ces grands hôtels sont tenus de mettre en place un comité d'entreprise (CE) dont les membres sont élus. Les CE ont pour missions de veiller à une certaine qualité du travail, au respect du droit ainsi que de constituer un CHSCT. L'une des actions des CE consiste aussi à fournir des prestations sociales et culturelles, comme l'illustre cet extrait d'entretien : "Je pense quion a l'un des meilleurs CE sur la place de Paris au niveau des avantages sociaux (...) On a beaucoup de spectacles proposés, on peut faire des commandes pour Noël à des prix imbattables. Il y a aussi la fête des enfants. Cette année, c'était une journée en famille à Eurodisney. On nous propose des séjours de ski l'hiver. Il y a un voyage qui nous est proposé. Cette année c'est le Pérou. Il y a des week-ends, à Vienne, à Rome, à Venise, à Prague, etc.» (femme de chambre, 51 ans, palace)

\section{Encadré 3}

\section{Les formes habituelles d'exploitation du secteur de l'hôtellerie-restauration}

Pour mieux saisir la qualité des conditions de travail dont bénéficie le personnel d'entretien de l'hôtellerie de luxe, il faut rappeler ce que sont les conditions de travail habituelles de ce secteur. Les métiers de la propreté sont ceux pour lesquels, en France, les formes d'exploitation salariale sont les plus courantes : travail à temps partiel, contrat à durée déterminée, recours massif à l'intérim, salaire horaire très proche du SMIC (salaire minimum interprofessionnel de croissance), travail fréquent le soir, les week-ends et les jours fériés, évolution salariale et perspectives de carrière généralement inexistantes... Les femmes qui acceptent des conditions de travail aussi dégradées sont souvent dans des situations personnelles qui ne leur laissent pas le choix : beaucoup sont immigrées (du moins à Paris), sans diplôme reconnu, certaines sont séparées avec des enfants à charge (Testenoire, 2010). Comme le constate Isabelle Puech (2006), ce sont des femmes vulnérables, mal payées, peu considérées et qui sont utilisées par les employeurs comme un salariat d'ajustement. La recherche du coût minimum conduit aussi à accroître la charge de travail par employée, produisant un turn-over élevé et des accidents de travail et /ou maladies professionnelles fréquents. Ces femmes de chambre sont d'autant plus vulnérables aux exigences de leurs employeurs qu'elles n'ont souvent aucune culture salariale et qu'elles exercent dans de petites équipes, ce qui rend difficile la construction de formes de résistances collectives.

\subsection{Une pratique professionnelle de distinction}

La notion d'élite ne repose pas uniquement sur les différents avantages obtenus statutairement de par la taille de ces entreprises. L'accès au métier et la mobilisation de compétences rares ou particulières permettent aussi d'affiner l'analyse. 
Les parcours professionnels des femmes de chambre que nous avons rencontrées sont proches de ceux observés dans l'ensemble du secteur : beaucoup ont débuté dans le métier comme " extra " (intérimaire), la plupart a attendu plusieurs années avant d'obtenir un $\mathrm{CDI}$ et presque toutes sont issues de l'immigration.

Ce premier constat établi, des différences existent entre les salariés recrutés dans les années 90 et ceux qui l'ont été au cours des décennies suivantes. Lors de cette première période, si la proportion d'immigrés était déjà forte, cette immigration provenait principalement de pays d'Europe du Sud et d'Europe de l'Est. Ces femmes de chambres étaient socialement plus proches des classes moyennes que ne le sont les nouvelles recrues. Contrairement à ce qui s'observe aujourd'hui, elles disposent d'un capital culturel non négligeable ${ }^{4}$ qu'elles n'ont pas pu, à leur arrivée en France, faire reconnaître. Dès lors, accepter un emploi de femme de chambre dans un établissement prestigieux leur est apparu comme une voie honorable d'insertion dans le salariat même si, pour beaucoup d'entre-elles, il s'agissait d'un emploi temporaire. Souvent mariées à de petits entrepreneurs indépendants et ayant des enfants fréquemment diplômés de l'enseignement supérieur, ces salariés ont des parcours qui les écartent de ceux de l'hôtellerie plus classique. Par la suite, la difficulté à changer d'emploi et l'attrait des conditions salariales proposées expliquent pour partie leur volonté de s'inscrire dans une carrière ${ }^{5}$ dans l'hôtellerie de luxe : outre leur bonne maîtrise du français, beaucoup d'entre-elles ont suivi des cours d'anglais, voire d'informatique, dans le cadre de la formation continue proposée par leur entreprise afin de mieux satisfaire les demandes d'une clientèle internationale et d'éventuellement obtenir une promotion professionnelle (devenir responsable de formation, première femme de chambre, voire gouvernante).

Par ailleurs, la présence, au sein des palaces de lustres très imposants, d'un mobilier constitué d'essence de bois rares, de matériaux nobles mais fragiles - comme l'utilisation du marbre dans les salles de bain - ainsi que de nombreux tissus précieux, etc., conduit à rendre le travail des femmes de chambres et des valets plus complexe que dans les hôtels de chaîne. Néanmoins, aucun de ces établissements n’exige une qualification particulière au moment du recrutement et aucun titre ou diplôme ne constitue réellement cette activité en métier ${ }^{6}$. Cette absence de qualification à l'entrée est faiblement compensée par la formation reçue dans les établissements. En seulement trois jours de formation sur le terrain, une femme de chambre se voit confier un service identique à celui des titulaires. Si en

\footnotetext{
4 Plusieurs femmes de chambre que nous avons rencontrées ont un niveau scolaire proche du baccalauréat. 5 Les possibilités de carrière verticale sont faibles et limitées aux postes de première femme de chambre ou de gouvernante d'étage. La carrière est horizontale par le passage du statut de CDD à CDI, puis la possibilité de choisir les horaires de travail (journée plutôt que nuit, semaine plutôt que week-end) enfin leur étage, c'est-à-dire celui où elles estiment avoir les meilleures conditions de travail.

6 Des formations de niveau BEP (brevet d'études professionnelles) hôtellerie-restauration ou de CAP (certificat d'aptitude professionnelle) « services hôteliers " ou « employé technique de collectivités » existent, mais elles ne sont pas demandées pour accéder à ces emplois.
} 
2004, seulement le quart des salariés de l'hôtellerie ont suivi au moins une action de formation, les femmes de chambre que nous avons enquêtées ont très fréquemment bénéficié de stages ou de cours (anglais, informatique, gestes et postures, ...), ce qui les place dans une situation privilégiée. Cependant, ces actions ont davantage pour objectif d'assurer l'appropriation de la culture spécifique de ces établissements que de permettre un développement de compétences susceptible de déboucher sur une promotion hiérarchique.

Les qualités professionnelles des personnels de l'hôtellerie de luxe se manifestent surtout dans des dispositions qui, dans les établissements plus ordinaires, sont rarement prises en compte par leurs employeurs : l'exercice du métier dans un palace ou un hôtel de luxe s'exprime dans la présentation de soi. Expertes en propreté, les femmes que nous avons rencontrées portaient une tenue impeccable, leur apparence physique était soignée : cheveux noués, maquillage et port de bijoux discrets, usage de déodorant, port de chaussures fermées, etc. Ces qualités à la fois esthétiques et comportementales font écho au standing de la maison et sont par conséquent exigées par la direction des établissements. Mais, dans une profession où le relationnel représente une part non négligeable de l'activité, elles constituent aussi une des conditions minimales d'un échange avec une clientèle elle-même traditionnellement soignée et exigeante.

Ainsi, les qualités professionnelles attendues de ces femmes de chambre dépassent leur seule aptitude à accomplir correctement leur mission de nettoyage. Elles doivent également acquérir une hexis corporelle et un sens de la relation aux clients. Ces qualités constituent en quelque sorte la part " noble " de leur métier, qui les différencie de leurs consœurs employées dans des établissements plus modestes. Nous n’avons pas, de ce point de vue, enregistré de différences notables entre les personnels des palaces et ceux des hôtels de chaîne, les exigences des directions à l'égard des personnels étant comparables.

$\mathrm{Si}$, pour les plus anciennes, cette hexis était le plus souvent incorporée avant leur entrée dans ces établissements, la présentation de soi fait l'objet aujourd'hui de consignes davantage formalisées par des directives, souvent affichées dans les locaux réservés aux personnels. Ces attentes sont aussi fréquemment rappelées, comme en témoignent les injonctions d'une gouvernante générale que nous avons accompagnée dans les étages et qui demandait au personnel de " ne pas parler fort "; "de fermer sa blouse ", " de ne pas traîner les pieds" ou encore " de se tenir droit».

Les femmes de chambre rencontrées dans l'hôtellerie de luxe jouissent donc bien de conditions de travail qui les distinguent des personnels d'entretien de l'hôtellerie standard et qui les éloignent des codes comportementaux des catégories populaires. C'est ce qui permet de dire que ces personnels bénéficient d'un statut privilégié, et autorise à parler d'une élite de métier.

Pourtant, ce qui ressort des entretiens, ce ne sont pas des propos de satisfaction liés à ces conditions privilégiées mais plutôt le sentiment croissant d'une dévalorisation symbolique du métier. 


\section{Des personnels désenchantés}

Au cours de la première partie de ce texte, nous avons développé l'idée que les femmes de chambres et les valets constituent objectivement une élite au sein d'un métier assez peu visible.

Mais au-delà des particularités qui distinguent les palaces des grands hôtels de chaine, une même tendance à la généralisation de principes de management est clairement perceptible dans les deux catégories d'établissements. Cette mutation entraîne une évolution des conditions de travail des salariés qui est vécue subjectivement sur le mode du déclassement.

Nous avons en premier lieu rendu compte du discours de déploration que produit ce sentiment de déclassement, avant d'analyser, en second lieu, les dimensions objectives de dégradation des conditions de travail de ces personnels.

\subsection{La nostalgie d'un âge d'or du métier ou le sentiment d'une déqualification}

Le processus de codification et de quantification du travail est l'une des réponses aux difficultés de remplissage que traversent les établissements de luxe. Pour ces hôtels, les taux de remplissage sont devenus l'urgence de ces dernières années ; cela les a incités à adopter une politique commerciale plus dynamique et une politique d'accueil des clients moins sélective.

Nos observations effectuées dans le hall d'accueil ou dans la salle du petit déjeuner ainsi que les propos rapportés par le personnel d'étage, conduisent à penser qu'il s'agit là d'une clientèle plus opportuniste cherchant à «profiter » des prestations offertes.

Aux grandes fortunes francophones, européennes ou américaines se sont juxtaposées celles du Golfe Persique, de Russie ou d'Extrême-Orient. Dans les palaces, ces nouveaux clients s'installent en délégation de parfois plus de 100 personnes et entretiennent avec le personnel d'étage des relations qui sont mal vécues par certaines femmes de chambre. Les nouvelles clientèles qui fréquentent les palaces viennent avec leur propre personnel : gouvernantes, femmes de chambre et même parfois cuisiniers. Ceci a pour effet d'éloigner le personnel des hôtels du " client » proprement dit car ils n'ont plus affaire qu'aux gouvernantes et femmes de chambre de la délégation. Elles ont perdu ce que l'on pourrait appeler la relation $a u$ client, pour une relation avec les employés $d u$ client. Dès lors, les rapports sociaux sont différents puisqu'il s'agit uniquement d'être au service d'un personnel de qualification équivalente. Les personnels hôteliers le perçoivent comme une déqualification puisque leur travail est confié à d'autres. Par ailleurs, l'absence de relation au client est 
aussi perçue comme une forme de non-reconnaissance : l'invisibilité étant dans ce cas-là quasiment totale :

"Quand un client me demande un bloc ben, je vais lui en donner deux ou trois, j'ai envie d'être généreuse pour des clients comme ça. Il me demande deux stylos, je lui en donne quatre. Mais c'est pas pour avoir des pourboires, je vous dis la vérité parce que les pourboires on les a quand on s'y attend pas, là c'est vraiment quand je trouve les clients gentils, souriants parce qu'il y a maintenant tellement de clients où on est invisible. Qui ne nous parlent pas, qui ne nous regardent pas. On dit "bonjour monsieur", il nous regarde pas, on est invisible, qu'est-ce que vous voulez faire? On les insulte un petit peu dans notre tête mais on reste avec le sourire "au revoir messieurs dames. " (Femme de chambre, 55 ans, palace)

Dans les grands hôtels de chaîne, une clientèle socialement plus modeste, attirée par les promotions offertes pour assurer le remplissage de l'établissement, transforme aussi la perception de la relation avec le client.

Dans les deux types d'établissement, les salariés les plus anciens expriment une nostalgie de l' " avant ": d'un travail différent basé sur une relation de service plus gratifiante. Propos qui laissent entrevoir le temps révolu d'une relation enchantée au travail :

"Je peux vous dire qu'à l'époque on avait beaucoup de temps. On avait le temps de s'occuper de nos clients, de discuter avec eux, de les aider. Non on n'avait pas moins de chambres à faire. On avait même peut-être plus de travail... Je ne sais pas. Il y a des choses qui sont disparues, il $y$ a des choses qu'on faisait à l'époque qu'on ne fait plus maintenant. Il y a des choses par contre qu'on ne faisait pas à l'époque et que l'on fait maintenant. Mais il y avait... Est-ce que c'est une organisation, est-ce que c'est... On avait le temps, on avait le temps. On était calmes, on était... Je ne peux pas vous expliquer parce que souvent moi aussi je me pose la question et je n'arrive pas à comprendre. " (Femme de chambre, 57 ans, palace)

Ce discours est logiquement plus fortement exprimé dans les palaces que dans les hôtels de chaîne où la clientèle a toujours été plus rarement issue des sphères sociales les plus fortunées (Wagner, 2007). Pour les personnels des palaces, entrer dans les chambres des clients, c'est-à-dire au sein même de leur intimité, les amenait à côtoyer un milieu social qui avait l'habitude d'être servi. Plusieurs ouvrages ont déjà été consacrés aux relations qui se tissent entre le personnel de service et les grands de ce monde (de Keyzer, 1997 ; Martin-Huan, 1997 ; Gutton, 1981 ; Martin-Fugier, 1997). F. Gutton souligne les conséquences culturelles de cette proximité : "La cohabitation permanente, la présence, de nuit comme de jour, des servantes à la cuisine ou des valets dans l'antichambre mettaient les domestiques au fait des secrets familiaux (...) Les domestiques bénéficient ainsi des privilèges attachés à la maison qui les emploie " "Maîtres et serviteurs ont même certaines formes de sociabilité commune". L'analogie entre le palace et la demeure bourgeoise ou aristocratique a certes des limites, mais on y retrouve certaines ressemblances, notamment dans l'exigence de la discrétion, indissociable de la part d'intimité que suppose la relation de service. 
Ainsi, les femmes de chambre des palaces ne se considèrent pas, à l'inverse de leurs collègues employées dans d'autres établissements moins prestigieux, comme faisant uniquement le "sale boulot ". Elles se présentent plutôt comme des employées de maison, c'est-à-dire comme des personnes ayant un nom et un visage auprès de ceux qu'elles ont l'habitude de servir :

"C'était très famille, très naturel avec la clientèle. Les clients nous disaient "on se sent chez nous, comme à la maison" Il y a des clients qui voulaient être au jeme parce qu'il y avait Brigitte, des clients qui voulaient être au $2^{i m e}$ parce qu'il y avait Sylvia [...] C'étaient des gens très discrets, qui ne demandaient presque jamais rien, des gens qui vont à peine toucher leur lit quand ils vont dormir, leurs affaires sont très bien rangées, ils sont très soignés, très classe. " (Femme de chambre, 56 ans, palace)

Ces relations de proximité lentement installées avec la clientèle traditionnelle semblent invalidées par la présence de nouveaux clients. Selon les témoignages recueillis, ces derniers font au mieux comme si les personnels d'étages n'existaient pas et les ramènent à leur stricte fonction de nettoyage.

Même si cette nostalgie de "l'avant " s'exprime avec moins d'intensité dans les hôtels de chaîne, elle existe aussi et se décline plus prosaïquement à propos de la question des pourboires :

"Ah non, ça fait rien du tout de salaire, dans la semaine, si vous avez 10, 15 euros c'est tout. Autrefois c'était plus ... Oui, surtout les américains, ils venaient beaucoup, j'avais souvent 100 francs, comme ça, le client il arrivait, il donnait 50 francs, 100 francs. " (Femme de chambre, 51 ans, hôtel de chaîne)

Dans les palaces, la question des pourboires est également évoquée, mais en plus de leur valeur monétaire, c'est aussi leur valeur symbolique qui change avec la nouvelle clientèle. Ce qui se donnait plutôt individuellement, de la main à la main, selon une relation de proximité (et peut être d'allégeance) semble être distribué, dans le cas des délégations, collectivement. Certains témoignages font état de largesses importantes de la part des délégations, en particulier celles du Moyen-Orient. Selon ce qui nous a été rapporté, "l'émir " fait donner par un de ses proches une enveloppe qui est ensuite redistribuée à l'ensemble du personnel, chacun recevant une part égale. Cet argent sans visage témoigne d'un certain rapport distant ; un argent anonyme, qui n'a plus de sens qu'en lui-même, tel que Georg Simmel (1988) avait pu le souligner et qui participe à une sorte de déshumanisation du travail.

\subsection{Standardisation et redéfinition du métier de femme de chambre}

Ce discours sur la déshumanisation du travail renvoie aussi à la diffusion plus générale d'outils de gestion dont les effets ont été à plusieurs reprises critiqués (Salais 2010). Dans le champ de l'hôtellerie-restauration, Sylvie Monchatre (2010) a notamment décrit le pro- 
cessus de rationalisation à l'œuvre dans l'hôtellerie contemporaine : "Les hôtels sont conçus sur le même modèle, les prestations sont uniformisées (...) les métiers d'accueil sont soumis à une rationalisation de l'interaction qui se traduit par des protocoles de conduite à tenir face au client (...) Ce client est d'ailleurs transformé en prescripteur doté d'instruments d'évaluation de la qualité de service qu'il est en droit d'exiger (...) Servir dans ces conditions consiste avant tout à " tenir la marque ", c'est-à-dire à se conformer en toutes circonstances au niveau de qualité affiché par l'entreprise."

La rationalisation des services et des tâches est certes plus ancienne et plus développée au sein des hôtels de chaîne, mais est désormais clairement présente dans les palaces ${ }^{7}$. Cette importation du management moderne est vécue comme une nouvelle contrainte et comme un appauvrissement du métier :

"Les nouvelles consignes, oui ça oblige à travailler différemment... on doit dire les mêmes phrases aux clients, on répond la même chose et ça nous déplait, ça déplait à tout le monde parce que c'est justement ça la différence ici, c'est qu'ici il y avait une ambiance que l'on ne trouvait pas ailleurs. Maintenant, j'ai bien peur que la mise en place des standards, ça crée une distance avec le client. " (Gouvernante générale, 48 ans, palace)

Le nouveau management qui accompagne le mouvement de restructuration /concentration de l'industrie hôtelière se traduit, sur le terrain, par un souci constant d'évaluation des résultats et des performances en regard d'objectifs (Durand, Le Floch, 2006). De ce point de vue, le travail des femmes de chambre est directement impacté. Faisant suite à la venue d'experts chargés d'observer le travail dans les chambres, de nouvelles procédures ont été " proposées ». A l'instar de ce qui se passe dans d'autres secteurs d'activité (Stewart et alii, 2006), un crédit de temps a été calculé pour chacune des différentes tâches à accomplir. Ainsi, en situation normale, faire une salle de bain est créditée de douze minutes, refaire entièrement le lit de dix, passer l'aspirateur dans une chambre standard de six. Dans les hôtels de chaîne une " recouche " compte pour 20 minutes de temps de travail, un départ pour trente cinq minutes, etc.

Afin d'accompagner ces transformations de l'activité de travail, des procédures d'implication du personnel on été mises en place comme, par exemple, "les commissions femmes de chambre $»^{8}$ où elles sont incitées à donner leur avis. Ces commissions, dans lesquelles sont invités les cadres, n'abordent jamais les questions de fond : celui du chronométrage des tâches et de l'extension, au sein de l'hôtellerie, des principes du " juste à temps ». Dans l'un des hôtels de chaîne dans lequel nous étions, la question du jour était " comment passer des couvertures à la couette ?"; dans un autre, la commission s'employait à pro-

7 Notamment parce qu'ils font maintenant pratiquement tous partie de multinationales. Le palace A a été racheté par une grande multinationale, l'enseigne B est présente dans trois grandes capitales dans le monde. 8 Les « commissions de femmes de chambre » ont pour fonction de faire échanger les personnels autour des difficultés rencontrées afin de les faire remonter à l'équipe de direction ainsi que de mutualiser les « trucs et astuces » qui permettent de faciliter le travail. 
diguer " les bons gestes et les bonnes postures de travail ». Afin de faciliter l'introduction des nouvelles procédures, certaines femmes de chambres se sont vu proposer un poste de " première femme de chambre ", dont l'une des missions est d'aider à l'application des "bonnes méthodes de travail». Les directions des hôtels sont soucieuses des risques que peuvent entraîner, pour leur personnel, l'exercice plus intensif d'un travail physiquement éprouvant. En effet, les maladies professionnelles, les arrêts de travail, etc., désorganisent les services et engendrent un coût pour l'entreprise. D'où de nouvelles préconisations et des séances de formation visant à faire acquérir aux femmes de chambre des gestes techniques potentiellement moins traumatisants ${ }^{9}$. Pourtant, si les directions du personnel de ces hôtels prennent en compte les demandes des femmes de chambre, tout se passe comme si les améliorations procédurales liées à l'exercice du métier de nettoyage ne parvenaient pas à contrebalancer la perte de sa dimension relationnelle.

En effet, la réorganisation des tâches et la gestion comptable de la relation avec la clientèle se traduisent par une augmentation du personnel d'encadrement intermédiaire qui n'est aujourd'hui plus issu du rang. Pour la direction des établissements, l'emploi de jeunes diplômées à ces postes est cohérent avec le processus de standardisation et de recherche de rentabilité dans lequel elle est engagée (Mahieu, 2007). A la différence des femmes de chambre, ces personnels ont été formés aux principes de management moderne et ils y adhèrent. En outre, ils ne sont pas porteurs de l'histoire de l'établissement et ne peuvent donc pas s'approprier le discours de déploration d'un passé mythifié. Mises à part les différences d'âge, de formation et, souvent, de milieu social, le recrutement de personnels plus diplômés contribue à remettre en cause la façon de penser et de faire le métier de femme de chambre. En effet, les valeurs et les références au travail ne sont pas les mêmes. D’un côté, c'est l'expérience accumulée et le savoir-faire, la bonne connaissance de la culture d'établissement et des clients qui sont mis en avant ; de l'autre, ce sont les savoirs formalisés et une approche plus organisationnelle et comptable qui sont privilégiés.

Cette réorganisation du travail a contribué à appauvrir le métier de femme de chambre en le vidant de son contenu relationnel au profit des gouvernantes. Des résistances apparaissent cependant. En particulier de la part du personnel le plus ancien qui adopte une posture non-coopérative. Ces gouvernantes, et plus particulièrement les plus jeunes, rencontrent alors des difficultés de coordination et d'organisation du travail :

"Vous avez une petite gouvernante qui arrive, c'est un calvaire pour elle, parce qu'ils vont être durs, très durs même avec elle, ils vont la tester. C'est très dur de gérer des gens qui ont trente ans de maison. Les gouvernantes ont souvent des problèmes à faire revenir les femmes de chambre

\footnotetext{
9 Dans les hôtels de chaîne, l'introduction de housses de couettes à la place des traditionnelles couvertures a fait l'objet de plusieurs cessions de formation. Par ailleurs, les lits sont munis d'un levier à pédale permettant de les rehausser afin d'éviter les efforts lombaires. L'attention portée par les directions des ressources humaines des grands hôtels aux pathologies professionnelles tranche avec une attitude plutôt désinvolte dans les autres établissements, si l'on s'appuie sur les observations réalisées par Emmanuelle Lada (2009).
} 
dans les chambres parce quielles ont peur. Parce quil faut voir aussi que beaucoup de femmes de chambre ou de valets ont du caractère, voire beaucoup de caractère! Donc quand vous avez une petite jeune qui a vingt trois ans et une femme de chambre qui est là depuis vingt ans et qui a quarante ans, c'est pas la petite jeune qui va lui apprendre à travailler!" (Gouvernante Générale, 44 ans, hôtel de chaîne)

L'introduction du " crédit temps » n'est pas sans conséquence sur l'exercice du métier. Des femmes de chambres ont évoqué une fatigue au travail qu'elles n'éprouvaient pas avant. Certes, les personnes vieillissent mais la fatigue éprouvée au travail est certainement un des effets de son intensification (Dejours, 2000) :

"C'est le rendement, le rendement, le rendement. Au niveau du travail il y a plus de pression. Il y a beaucoup plus de pression, beaucoup plus de chiffres. Avant ce n'était pas comme ça, pas dans ce genre d'établissement. Moins en tout cas. " (Femme de chambre, 56 ans, palace)

Les principes de rationalisation et la recherche constante de gains de productivité rendent aussi plus difficile la relation de service avec le client :

"Une fermme de chambre, elle n'a plus le temps pour le service, pour défaire les valises par exemple. Les fermmes de chambre n'osent plus proposer ces services parce que si elles passent un quart d'heure avec un client, elles vont perdre du temps sur leur travail et au bout y'aura une remarque de la gouvernante et si ça se reproduit, un entretien avec la gouvernante générale. " (Femme de chambre, 48 ans, palace)

\section{Conclusion}

Ainsi, nous nous étions fixés pour projet de recherche d'identifier les caractéristiques d'une élite professionnelle invisible ; cependant, l'enquête nous a conduit à repérer un processus de déclassement induit par les effets des nouvelles techniques managériales qui rendent secondaires certaines des compétences professionnelles qui constituaient la partie la plus noble du métier.

Sur le terrain particulier de l'hôtellerie de luxe, ce déclassement n'apparait pas évident de prime abord. Les conditions de travail ainsi que les rémunérations perçues par les femmes de chambre et les valets restent encore aujourd'hui significativement plus intéressantes que dans le reste du secteur hôtelier. Ces salariés bénéficient d'un statut défendu par des délégués syndicaux et des représentants du personnel. Les directions générales et les DRH veillent à associer les personnels aux changements organisationnels présentés comme incontournables.

Cependant, la codification liée aux impératifs du new management, l'accroissement du personnel d'encadrement, transforment le métier de femme de chambre et tendent de plus en plus à réduire les salariés à de simples techniciens de propreté, sans autonomie ni marge de manœuvre. C'est pourquoi on observe, dans les discours des personnes interrogées, 
un décalage entre les conditions objectives de travail et la perception des transformations subjectives du rapport au métier. Cette perception est plus marquée chez les femmes de chambre ayant le plus d'ancienneté et dans les palaces, où ces modifications sont plus importantes et plus récentes.

Lors des entretiens, même si les conditions de travail et leur transformation ont été largement évoquées, c'est sur la nouvelle clientèle que les propos les plus amers ont été tenus. Comme si les " nouveaux riches " et/ou les clients "à prix promotionnel " cristallisaient à eux seuls l'ensemble des frustrations accumulées depuis deux décennies. Comme si la relation vécue sur le mode du privilège et de l'exceptionnel, au contact d'une clientèle favorisée, qui leur faisait "partager " pour un instant une petite part de leur intimité, avait pour effet de les distinguer à leur tour comme l'élite du métier (Gutton, 1981 ; Pinto, 1990) tout en transformant en qualités professionnelles la modestie et l'humilité héritées de leurs origines sociales.

\section{Bibliographie}

Becker S. H. (2002), Les ficelles du métier, Paris, La Découverte.

Cartier M., Retière N., Siblot J. (2010), Le salariat à statut. Genèses et cultures, Rennes, PUR.

Castel R. (1995), Les métamorphoses de la question sociale, une chronique du salariat, Paris, Fayard.

Charle C. (1987), Les élites de la République. 1880-1900, Paris, Fayard.

De Keyzer D. (1997), Madame est servie. Vivre au service de la noblesse et de la bourgeoisie (1900-1995), Bruxelles, La Longue Vue.

Dejours C. (2000), Travail, usure mentale. Essaie de psychopathologie du travail, Paris, Bayard.

Durand J.-P., Le Floch M.-C. (2006), La question du consentement au travail. De la servitude volontaire à l'implication contrainte, Paris, L'Harmattan.

Guégnard C. (dir.) (2004), A la recherche d'une conciliation des temps professionnels et personnels dans l'hôtellerie-restauration, Céreq Relief, 7, échanges du Cereq.

Guégnard C. et Mériot S.-A., (2007), «Régulation et précarisation : l'exemple des femmes de chambres ", Communication au XI journées internationales de sociologie du travail, Londres 20-22 juin 2007.

Guégnard C., Mériot S.-A. (2009), "Alice au pays des hôtels : de l'autre côté du miroir ", in Bas salaires et qualité de l'emploi : l'exception française ?, Caroli E., Gautié G., collection du CEPREMAP, Presses de l'École normale supérieure. 
Gutton J.-P. (1981), Domestiques et serviteurs dans la France de l'Ancien Régime, Paris, Aubier-Montaigne.

INSEE (2003), "L'hôtellerie, la restauration et les cafés, un secteur très spécifique en termes d'emploi et de rémunération ", $n^{\circ} 889$, mars.

Lada E. (2009), « Division du travail et précarisation de la santé dans le secteur hôtelier en France : de l'action des rapports sociaux de sexe et autres rapports de pouvoir ", Travailler, $\mathrm{n}^{\circ} 22$, pp. 9-26.

Mahieu C. (2007), "Le management intermédiaire en transformation ", Revue française de gestion, $\mathrm{n}^{\circ} 172$, pp. 49-61.

Martin-Fugier A. (2004), "La place des bonnes : la domesticité féminine à Paris en 1900 », Tempus.

Martin-Huan J. (1997), La Longue Marche des domestiques en France du XIX siècle à nos jours, Nantes, Éditions Opéra.

Monchatre S. (2010), Etes-vous qualifiée pour servir?, Paris, La Dispute.

Pigenet M. (1990), "A propos de l'aristocratie ouvrière. Elites professionnelles et militantes au XIXe siècle dans le département du Cher ", Romantisme, n 70, pp. 91-102

Pinçon M. \& Pinçon-Charlot M. (1996), Grandes Fortunes. Dynasties familiales et formes de richesse en France, Paris, Payot.

Pinto J. (1990), "Une relation enchantée », Actes de la recherche en sciences sociales, vol. 48, septembre, pp. 32-48.

Puech I. (2006), « Femmes et immigrés : corvéable à merci », Travail, genre et société, ${ }^{\circ} 16$.

Salais R. (2010), « La donnée n’est pas donnée. Pour une analyse critique de l'évaluation chiffrée de la performance ", Revue française d'administration publique, $\mathrm{n}^{\circ} 135$, pp. 497-515

Simmel G. (1988), Philosophie de l'argent, Paris, PUF.

Stewart P. et alii (2006), "Les ouvriers de Vauxhall face à la lean production", Le Mouvement Social, n 217 , pp. 33-52.

Testenoire A. (2010), «Articuler les paradigmes de la reconnaissance et de la redistribution ; la situation des femmes de chambre ", L’Homme et la société, n 176-177, pp. 83-99.

Vigarello G. (1985), Le propre et le sale. L'hygiène du corps depuis le Moyen-Age, Paris, Seuil.

Wagner A.-C. (2007), Les classes sociales dans la domination, Paris, La Découverte. 